\title{
Analysis of trinucleotide repetitive sequences for Korean patients with spinocerebellar ataxia types 8 , 12 , and 17
}

Gu-Hwan Kim', Sun Ju Chung ${ }^{2}$, Ho-Sung Ryu², Jaemin Kim', Jin-Joo Lee', Seoung Hoon Choi', Juyeon Lee', Beom Hee Lee', Jin-Ho Choi ${ }^{3}$, and Han-Wook Yoo ${ }^{1,3, *}$

${ }^{1}$ Medical Genetics Center, Asan Medical Center, ${ }^{2}$ Department of Neurology, Asan Medical Center, ${ }^{3}$ Department of Pediatrics, Asan Medical Center Children's Hospital, University of Ulsan College of Medicine, Seoul, Korea

Purpose: Spinocerebellar ataxias (SCAs) are progressive neurodegenerative disorders with diverse modes of inheritance. There are several subtypes of SCAs. SCA 8, SCA 12, and SCA 17 are the less common forms of SCAs with limited information available on their epidemiological profiles in Korea. The purpose of this study was to investigate the prevalence of SCA8, SCA12, and SCA17 in Korea.

Materials and Methods: Ninety-six unrelated Korean patients were enrolled and showed normal trinucleotide repeats through polymerase-chain reaction (PCR) for the genes $A T X N 1, A T X \mathrm{~N} 2, A T X \mathrm{~N} 3, C A C N A 1 A$, and $A T X \mathrm{~N} 7$, which correspond to SCA1, SCA2, SCA3, SCA6, and SCA7, respectively. PCR products from patients were further analyzed by capillary electrophoresis using fluorescence labeled primers for the genes $A T X N 8 O S, P P P 2 R 2 B$, and TBP, which correspond to SCA8, SCA12, and SCA17.

Results: Three patients had 104, 97, and 75 abnormal expanded repeats in the ATXN8OS gene, the causative gene for SCA8. None of the patients exhibited abnormal repeats in SCA12 and SCA17. Normal trinucleotide repeat ranges of the cohort in this study were estimated to be 17-34 copies (average, 24 \pm 4 copies) for SCA8, 7-18 copies (average, 13 \pm 3 copies) for SCA12, and 26-43 copies (average, 35 \pm 2 copies) for SCA17.

Conclusion: This study demonstrated that SCA8, SCA12, and SCA17 are rare in Korean patients with SCA, and further genetic studies are warranted to enhance the mutation detection rate in the Korean SCA population.

Key words: Spinocerebellar ataxia, Spinocerebellar ataxia 8, Koreans.

\section{Introduction}

Spinocerebellar ataxias (SCAs) are hereditary neurodegenerative disorders characterized by progressive ataxia, cerebellar dysarthria, spasticity, extrapyramidal signs, cognitive impairment, and epilepsy $[1,2]$. SCAs are divided into autosomal dominant, autosomal recessive, $\mathrm{X}$-linked, and mitochondrial SCAs according to the mode of inheritance. Among these, autosomal dominant SCAs is the most common subtype, and more than 30 autosomal dominant SCAs have been identified to date. The causative genes associated with SCAs include SCA1 (ATXN1), SCA2 (ATXN2), SCA3 (ATXN3), SCA5 (SPTBN), SCA6 (CACNA1A), SCA7 (ATXN7), SCA8

Received: 30 May 2015, Revised: 2 June 2015, Accepted: 8 June 2015, Published: 30 June 2015

*Corresponding author: Han-Wook Yoo, M.D., Ph.D.

Department of Pediatrics and Medical Genetic Center, Asan Medical Center, University of Ulsan College of Medicine, 88 Olympic-ro 43-gil, Songpa-gu, Seoul 138-736, Korea.

Tel: +82-2-3010-3374, Fax: +82-2-473-3725, E-mail: hwyoo@amc.seoul.kr

Conflict of interest: We declare that we do not have any conflicts of interests.

(c) This is an open-access article distributed under the terms of the Creative Commons Attribution Non-Commercial License (http://creativecommons.org/licenses/by-nc/4.0/) which permits unrestricted non-commercial use, distribution, and reproduction in any medium, provided the original work is properly cited.

(c) Copyright 2015 by the Korean Society of Medical Genetics

www.e-kjgm.org 
(ATXN80S), SCA10 (ATXN10), SCA11 (TTBK2), SCA12 (PPP2R2B), SCA13 (KCNC3), SCA14 (PKC), SCA17 (TBP), SCA19/22 (KCND3), SCA21 (TMEM240), SCA23 (PDYN), SCA25 (SCA25), SCA26 (EEF2), SCA27 (FGF14), SCA28 (AFG3L2), and SCA29 (ITPR1), and recent developments in the genetic field using next-generation sequencing techniques continue to reveal the new causative genes in SCAs [3,4]. SCA1, SCA2, SCA3, SCA6, and SCA7 are the most prevalent forms of SCA worldwide. However, only a few studies have been conducted in the Korean population [5-8]. Among these, SCA2, SCA3, and SCA6 are found to be the most common subtypes, followed by SCA7 and SCA1 [9]. However, in $70 \%$ of the patients, their SCA subtypes remain unidentified as routine screenings were only conducted for SCA1, SCA2, SCA3, SCA6, SCA7, and SCA13 [10]. With the exception of a few case reports, the prevalence of SCA8, SCA12, and SCA17 in the Korean population remains relatively unknown $[11,12]$.

This study was performed to investigate the prevalence SCA8, SCA12, and SCA17 in Korean patients with SCAs who were negative for SCA1, SCA2, SCA3, SCA6, and SCA7.

\section{Materials and Methods}

This study was approved by the Institutional Review Board of Asan Medical Center, Seoul, Korea. Ninety-six unrelated Korean patients (44 males and 52 females) with clinically suspected SCA who had normal trinucleotide repeat (TNR) copy numbers of SCA1 (ATXN1), SCA2 (ATXN2), SCA3 (ATXN3), SCA6 (CACNA1A), and SCA7 (ATXN7) were enrolled in this study. The mean age at enrollment was $53 \pm 10$ years.

Genomic DNA was isolated from peripheral blood leukocytes using the Gentra Puregene blood kit (Qiagen, Hilden, Germany). Genes for SCA1, SCA2, SCA3, SCA6, and SCA7 were tested as previously described [9].

To identify abnormal TNR expansion of SCA8 (ATXN80S), SCA12 (PPP2R2B), and SCA17 (TBP), we used 6-FAM labeled primer sets for $P C R$, followed by capillary electrophoresis. The primer sequences of SCA8, SCA12, and SCA 17 were designed using reference sequences from GenBank NT_024524.14, NT_029289.11, and NT_025741.15, respectively. The primer sequences, 5'-tttgagaaaggcttgtgagg actgagaatg-3' (sense) and 5'-ggtccttcatgttagaaaacctggct-3' (antisense), were used for SCA8, 5'-tgctgggaaagagtcgtg-3' (sense) and 5'-gccagcgcactcaccctc-3' (antisense) for SCA 12, and 5'-gaccccacagcctattcaga-3' (sense) and 5'-ttgactgctgaacggctgca-3' (antisense) for SCA17.

PCR was carried out with a final volume of $20 \mu \mathrm{L}$, containing
$100 \mathrm{ng}$ of template DNA, $1 \mu \mathrm{M}$ of each primer sets, $200 \mu \mathrm{M}$ of dNTP mixture, $1.5 \mathrm{mM}$ of $\mathrm{MgCl}_{2,} 50 \mathrm{mM}$ of $\mathrm{KCl}, 10 \mathrm{mM}$ of Tris$\mathrm{HCl}(\mathrm{pH} 8.3)$ and 1 unit of Taq polymerase (Promega, Madison, WI, USA). Genes were amplified for 30 cycles with the following parameters: denaturation at $94^{\circ} \mathrm{C}$ for $30 \mathrm{~s}$, annealing at $55^{\circ} \mathrm{C}$ for $30 \mathrm{~s}$, and reaction at $72^{\circ} \mathrm{C}$ for $45 \mathrm{~s}$ using the PTC-200 (MJ Research, Watertown, MA, USA). One microliter of PCR products was used for capillary electrophoresis with the ABI3130xI (Applied Biosystems, Foster, CA, USA). The reaction mixture also contained $0.5 \mu \mathrm{L}$ of GeneScan 500 Liz and $10 \mu \mathrm{L}$ of HiDi formamide (Applied Biosystems) in condition of POP7-polymer. The oven temperature was set at $60^{\circ} \mathrm{C}$, injection at $1.2 \mathrm{kV}$ for 20 $\mathrm{s}$, and the gel was run at $15 \mathrm{kV}$ for 1,200 s. Data were analyzed with GeneMapper v4.0 (Applied Biosystems).

\section{Results}

Among the 96 patients enrolled in this study, three were heterozygotes for the expanded TNR numbers in the ATXN80S gene, which is responsible for SCA8 (Fig. 1). None of the patients showed expanded TNR number in the PPP2R2B gene (SCA12) or the TBP gene (SCA17).

\section{Clinical and genetic features of three patients with SCA8 1) Case 1}

The patient was a 38-year-old female Korean who suffered from dysarthria since the age of 34 years. She also had dysphagia, but no ataxia or gait disturbance. Her mother and grandmother also had gait disturbance. Brain magnetic resonance imaging (MRI) showed prominent cerebellum folia. The genetic test for the ATXN8OS gene revealed that the patient had 104 (CTG.CAG)n copies (normal range, 5-50 copies) in one allele, with normal numbers (29 copies) of (CTG-CAG)n copies on the other allele.

\section{2) Case 2}

A 63-year-old female Korean was presented with ataxia, gait disturbance, dysarthria, and bradykinesia. A brain MRI revealed diffuse atrophy of the pons and the cerebellum (Fig. 2). She had been treated with quetiapine fumarate and fluvoxamine. She had expanded (CTG.CAG)n repeats (97 copies) in one allele of the ATXN8OS gene, while the other allele contained 17 copies of TNRs.

\section{3) Case 3}

A 67-year-old female Korean experienced progressive 

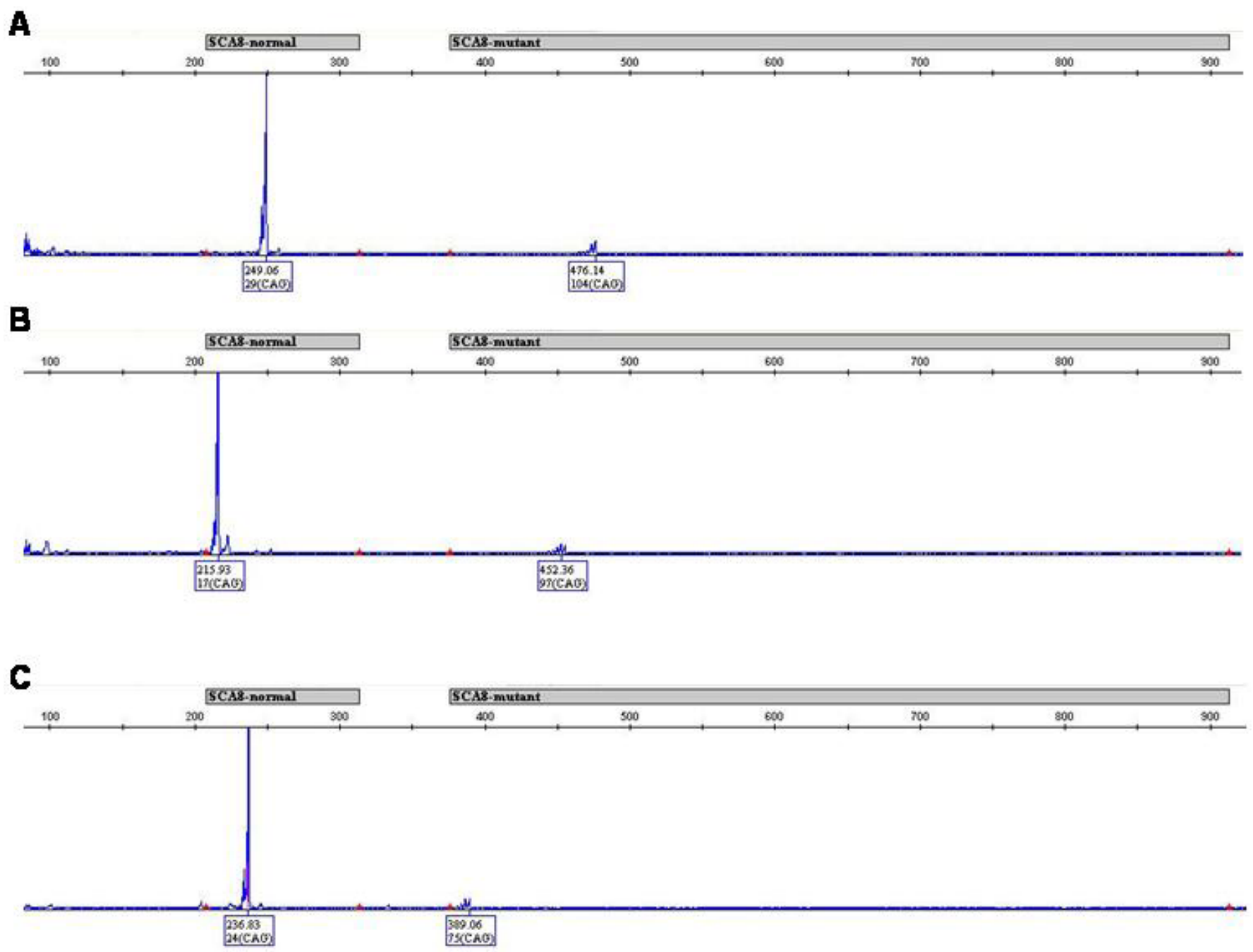

Fig. 1. GeneScan profiles of $A T X N 80 S$ alleles from three SCA8 patients. The peak indicates the ATXN80S allele of each patient. The upper gray bar indicates normal and mutant ranges of copy numbers of $A T X N 80 S$. The size of product (bp) and calculated copy numbers are listed in the boxes. Analyses were performed using GeneMapper 4.0 (Applied Biosystems, Foster, CA, USA). (A) Patient 1 has a mutant allele of 104 repeats with a normal allele of 29 repeats. (B) Patient 2 has a mutant allele of 97 repeats and a normal allele of 17 repeats. (C) Patient 3 has a mutant allele of 75 repeats and a normal allele of 24 repeats.

gait disturbance since 62 years of age. She had mild rigidity, bradykinesia, and dysmetria. Diminished metabolism in the cerebellum and midbrain was detected via brain positron emission tomography (Fig. 3). She had expanded (CTG · CAG) $\mathrm{n}$ repeats (75 copies) in one allele of the ATXN80S gene with normal number of TNRs (24 copies) in the other allele.

\section{Normal ranges of trinucleotide repeats in SCA8, SCA12, and SCA17}

The mean copy number of (CUG)n in the ATXN8OS gene, which was responsible for $\mathrm{SCA} 8$, were calculated in 93 patients and were found to be $24 \pm 4$ repeats (range, $17-34$ repeats). Data from the three patients with abnormal number of TNRs were excluded from the analysis. Of note, 17 repeats (29/186 alleles,
16\%), 23 repeats (23/186 alleles, 12\%), and 26 repeats (26/186 alleles, 14\%) were found as the common repeat numbers, comprising $42 \%$ of all the alleles tested. The mean copy number of (CAG)n repeats in the PPP2R2B gene, which was responsible for $\mathrm{SCA} 12$, was $13 \pm 3$ repeats (range, $7-18$ repeats) among the 96 patients tested. The mean copy number of (CAG.CAA)n repeat in the TBP gene, which was responsible for SCA17, was $35 \pm 2$ repeats (range, 26-34 repeats). We found that 9-15 repeats were common in SCA12, whereas 35 repeats was the most prevalent copy number in SCA17 (Fig. 4).

\section{Discussion}

SCAs are caused by one of the three genetic abnormalities. 


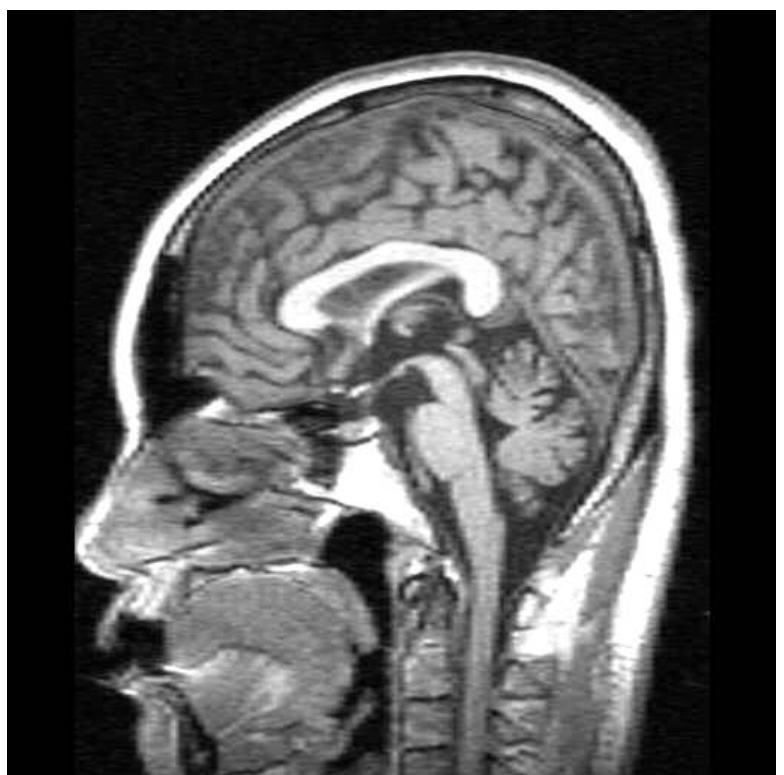

Fig. 2. Magnetic resonance image of patient 2. Diffuse atrophies of pons and cerebellum are noted.
The first is the abnormal expansion of a CAG trinucleotide in protein coding regions of specific genes. Out of all SCA subtypes, SCA1, SCA2, SCA3, SCA6, SCA7, SCA12, and SCA17 are caused by abnormal expansion of (CAG)n repeat, which leads to accumulation of toxic poly-glutamine tract. The normal ranges of $(C A G) n$ repeats of SCA1, SCA2, SCA3, SCA6, and SCA7 were 6-39 copies, 14-32 copies, 12-40 copies, 4-18 copies, and 7-18 copies, respectively. The mutant ranges of the TNRs in SCA1, SCA2, SCA3, SCA6, and SCA7 were 41-83 copies, 34-77 copies, 62-88 copies, 22-30 copies, and 38-200 copies, respectively [2]. The second genetic cause of SCA is trinucleotide expansion in the non-coding region like SCA8 and SCA10. The last genetic cause is point mutation in the coding region of morbid genes such as SCA5 (SPTBN), SCA11 (TTBK2), SCA13 (KCNC3), SCA14 (PKC), SCA19/22 (KCND3), SCA23 (PDYN), and more [1].

The common disease manifestation of SCA is progressive neuro-degeneration, but SCA also shows a wide phenotypic spectrum, and its severity is associated with the copy number

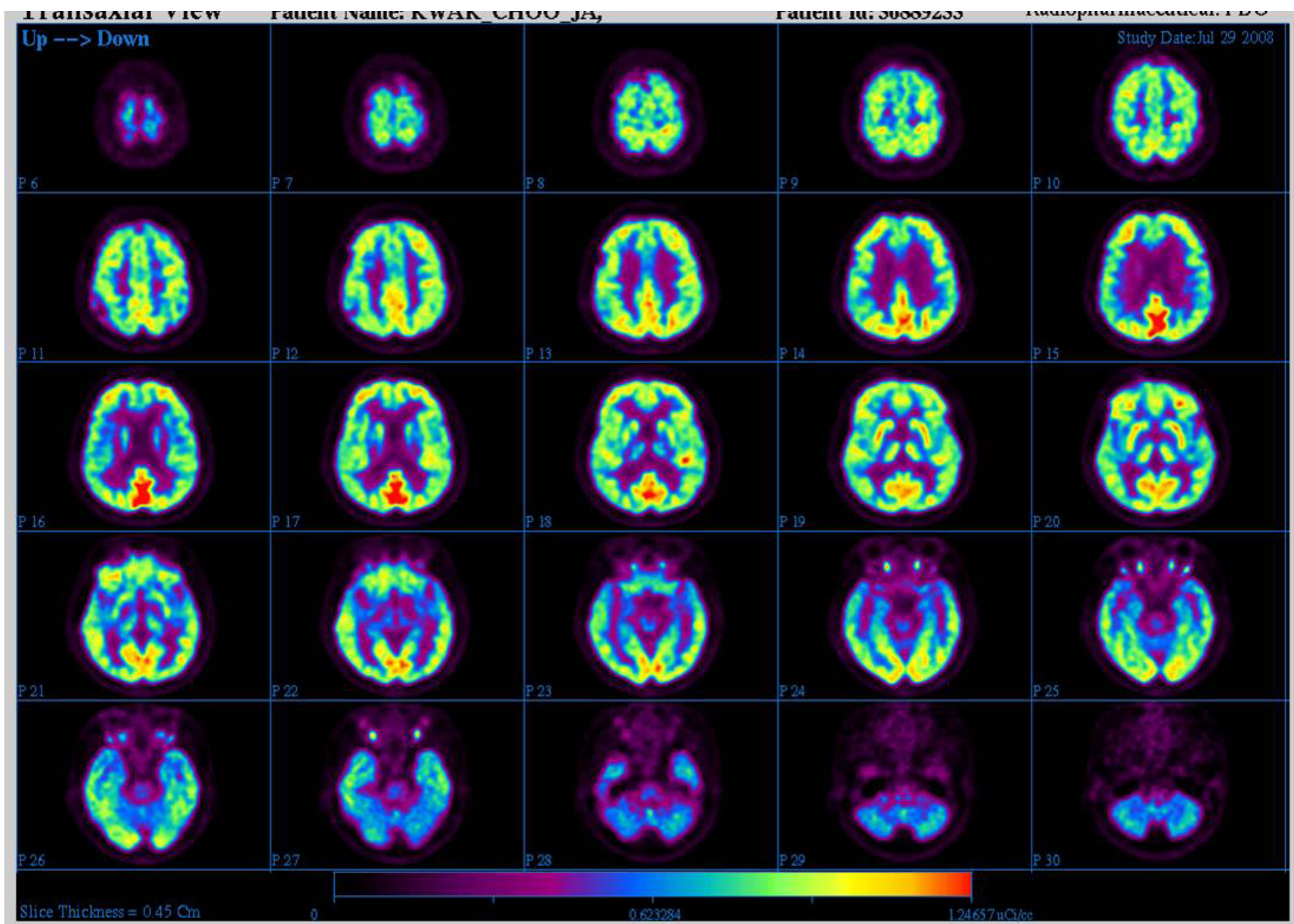

Fig. 3. Brain positron emission tomography of patient 3. Decreased metabolisms are noted in the cerebellum, the midbrain, and the pons. 

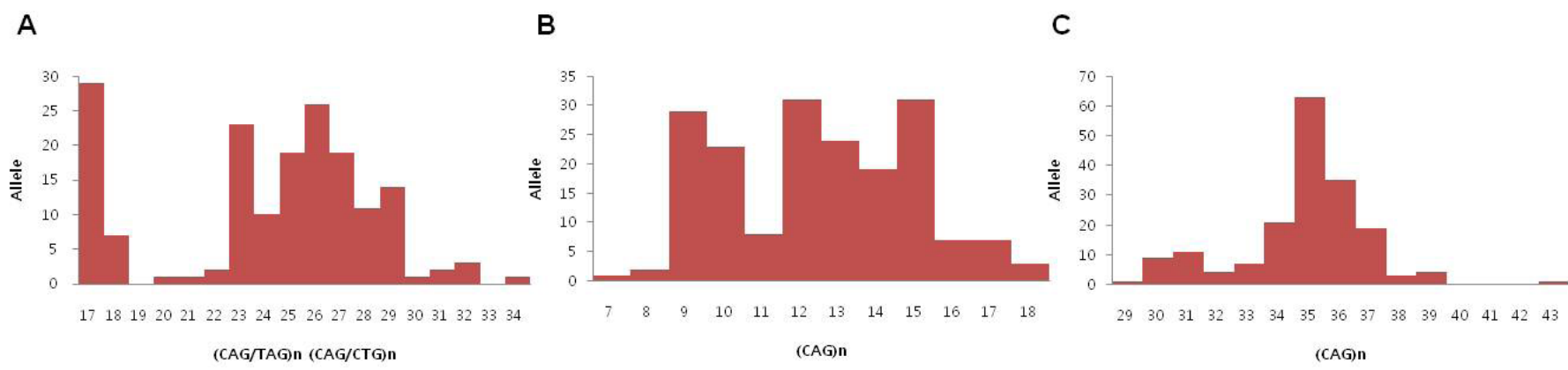

Fig. 4. Distribution of trinucleotide copy numbers of SCA8, 12, and 17 in Korean patients. Normal ranges and distributions of TNRs in SCA8 (A), SCA12 (B), and SCA17 (C).

size of expanded repetitive nucleotides. Patients with a larger expansion manifest SCA at an earlier age, whereas those with a smaller expansion have late onset-age [4]. In the genetic abnormality of TNR expansions, the expansion size of the TNR is correlated with patient's prognosis. Thus, it is important to determine the copy numbers of (CAG)n in these causative genes. To date, the relationship between expansion size and diseaseonset age of SCA1, SCA2, SCA3, SCA6, and SCA7 have been reported in many studies [9], but, in the case of SCA8, SCA10, SCA12, and SCA17, limited information is available.

Tests for the frequent subtypes, SCA1, SCA2, SCA3, SCA6, and SCA7 are commercially available and are done as a panel tests, whereas tests for the infrequent subtypes, SCA8, SCA10, SCA11, SCA12, SCA13, SCA14, SCA17, SCA27 are usually done individually or as a multi-gene test for the patients who are negative for the frequent subtypes [3]. In our study, the 96 patients enrolled were all negative for SCA1, SCA2, SCA3, SCA6, and SCA7, and the results SCA8, SCA12, and SCA17 tests indicate that these subtypes are very rare, where only three patients were positive for SCA8.

SCA8 is caused by the expansion of (CTA·TAG)n(CTG.CAG) $\mathrm{n}$ repeats in 3 '-untranslated region of the $A T X N 80 S$ gene. Its normal repeat number ranges from 15 to 50 copies, while mutants range from 80 to 250 copies. Recently, SCA8 also known to be caused by (CAG)n repeats in the open reading frame of the ATXN8 gene, an overlapping gene of ATXN8OS. Although the mechanism is not well defined, SCA8 is presumed to occur due to accumulation of toxic RNAs of ATXN8OS like as RNA toxicity by (CTG)n TNR expansion of DMPK in myotonic dystrophy type I $[13,14]$ or due to abnormal polyglutamine expansion of ATXN8 that results from expansion of bidirectional TNRs complementary to the ATXN8 and ATNX8OS genes [15]. Of note, reduced penetrance has been observed even in subjects with mutant-sized allele ranging from 80 to 250 copy numbers.
It should be noted that even subjects with a huge expansion of over 500 copies may not necessarily show ataxia [16]. In this respect, case 3 in the current report had a copy number of 75, which was smaller than the mutant copy numbers, and her cerebellar ataxic manifestation may be explained by high penetrance.

Anticipation is a major issue that should be discussed during genetic counseling with families that have a history of SCA. In case of SCA8, the repeat size is always expanded when it is inherited maternally, whereas it is shortened when paternally inherited [16].

We calculated the normal range of the TNRs in patients who were negative for SCA8, SCA12, and SCA17. The number of TNRs from these patients was within the normal ranges as previously reported, and some common allele sizes were noted in each subtype. The normal copy numbers of SCA8, 12, 17 in Korean population ranged from 17 to 34 (mean $24 \pm 4$ ), 7 to 18 (mean $13 \pm 3$ ), and 26 to 43 (mean $35 \pm 2$ ), respectively. These numbers were similar to previously reported range of $15-50,4-32$, and 25-42 for SCA8, 12, and 17, respectively.

In conclusion, we have identified three SCA8 patients out of 96 unrelated patients with clinically suspected SCA who showed normal TNR copies in SCA1, 2, 3, 6, and 7. There were no patients with SCA12 and 17. Further study should be performed to confirm the prevalence of SCA subtypes as it would provide valuable information to generate gene panel tests for rapid diagnosis of patients who show indications of SCAs in Korea.

\section{Acknowledgements}

This research was supported by a grant from the National Research Foundation of Korea and funded by the Ministry of Education, Science, and Technology (grant No. 2011-0019674). 


\section{References}

1. Dueñas AM, Goold R, Giunti P. Molecular pathogenesis of spinocerebellar ataxias. Brain 2006;129:1357-70.

2. Schöls L, Bauer P, Schmidt T, Schulte T, Riess O. Autosomal dominant cerebellar ataxias: clinical features, genetics, and pathogenesis. Lancet Neurol 2004;3:291-304.

3. Bird TD. Hereditary ataxia overview. In: Pagon RA, Adam MP, Ardinger $\mathrm{HH}$, Wallace SE, Amemiya A, Bean $\sqcup H_{\text {, et al., eds. GeneReviews }}{ }^{\circledR}$ [Internet]. Seattle (WA): University of Washington, 1993-2015. [http:// www.ncbi.nlm.nih.gov/pubmed/20301317].

4. Paulson HL. The spinocerebellar ataxias. J Neuroophthalmol 2009;29: 227-37.

5. Jin DK, Oh MR, Song SM, Koh SW, Lee M, Kim GM, et al. Frequency of spinocerebellar ataxia types 1,2,3,6,7 and dentatorubral pallidoluysian atrophy mutations in Korean patients with spinocerebellar ataxia. J Neurol 1999;246:207-10.

6. Kim JY, Park SS, Joo SI, Kim JM, Jeon BS. Molecular analysis of spinocerebellar ataxias in Koreans: frequencies and reference ranges of SCA1, SCA2, SCA3, SCA6, and SCA7. Mol Cells 2001;12:336-41.

7. Bang OY, Huh $K_{1}$ Lee PH, Kim HJ. Clinical and neuroradiological features of patients with spinocerebellar ataxias from Korean kindreds. Arch Neurol 2003;60:1566-74.

8. Jeong SY, Jang SH, Kim HJ. Spinocerebellar ataxia 7 (SCA7). J Genet Med 2007;4:22-37.
9. Choi IH, Kim GH, Lee BH, Choi JH, Yoo HW. Distribution of five common subtypes of spinocerebellar ataxia in the Korean population. J Genet Med 2014;11:69-73.

10. Lee $W Y$, Jin $D K$, Oh MR, Lee JE, Song SM, Lee EA, et al. Frequency analysis and clinical characterization of spinocerebellar ataxia types 1 2, 3, 6, and 7 in Korean patients. Arch Neurol 2003;60:858-63.

11. Lee $\mathrm{SH}, \mathrm{Ki}$ CS, Cho HI, Lee PW, Kim JW, Lee WY. A case of familial spinocerebellar ataxia type 8. J Korean Neurol Assoc 2004;22:659-62.

12. Choi JM, Woo MS, Kim S, Ma HI, Sung YH, Lee PH, et al. Trinucleotide repeats number in SCA2, SCA3, and SCA17 in early-onset Parkinson's disease. J Korean Neurol Assoc 2008;26:23-7.

13. Ikeda Y, Shizuka-Ikeda M, Watanabe M, Schmitt M, Okamoto K, Shoji M. Asymptomatic CTG expansion at the SCA8 locus is associated with cerebellar atrophy on MRI. J Neurol Sci 2000;182:76-9.

14. Ranum LP, Cooper TA. RNA-mediated neuromuscular disorders. Annu Rev Neurosci 2006;29:259-77.

15. Moseley ML, Zu T, Ikeda Y, Gao W, Mosemiller AK, Daughters RS, et al. Bidirectional expression of CUG and CAG expansion transcripts and intranuclear polyglutamine inclusions in spinocerebellar ataxia type 8. Nat Genet 2006;38:758-69.

16. Ayhan F, Ikeda Y, Dalton JC, Day JW, Ranum LPW. Spinocerebellar ataxia type 8. In: Pagon RA, Adam MP, Ardinger HH, Wallace SE, Amemiya $A$, Bean $\sqcup H$, et al., eds. GeneReviews ${ }^{\circledR}$ [Internet]. Seattle (WA): University of Washington, 1993-2015. [http://www.ncbi.nlm. nih.gov/books/NBK1268/] 